27

$8^{2}$

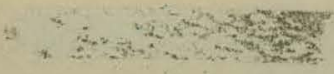

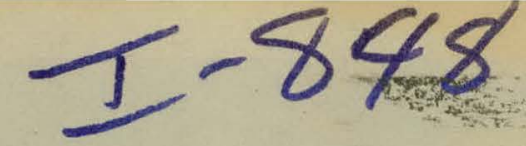

i. SANDIA REPORT SAND81-2476 • Unlimited Release

$\{$ Printed November 1981

24tontis

\title{
Remote Job Entry (RJE) for VAX/VMS User Guide
}

C. Douglas Brown, Norman H. Samuelson

Prepared by

Sandia National Laboratories

Albuquerque, New Mexico 87185 and Livermore, California 94550

for the United States Department of Energy

under Contract DE-AC04-76DP00789

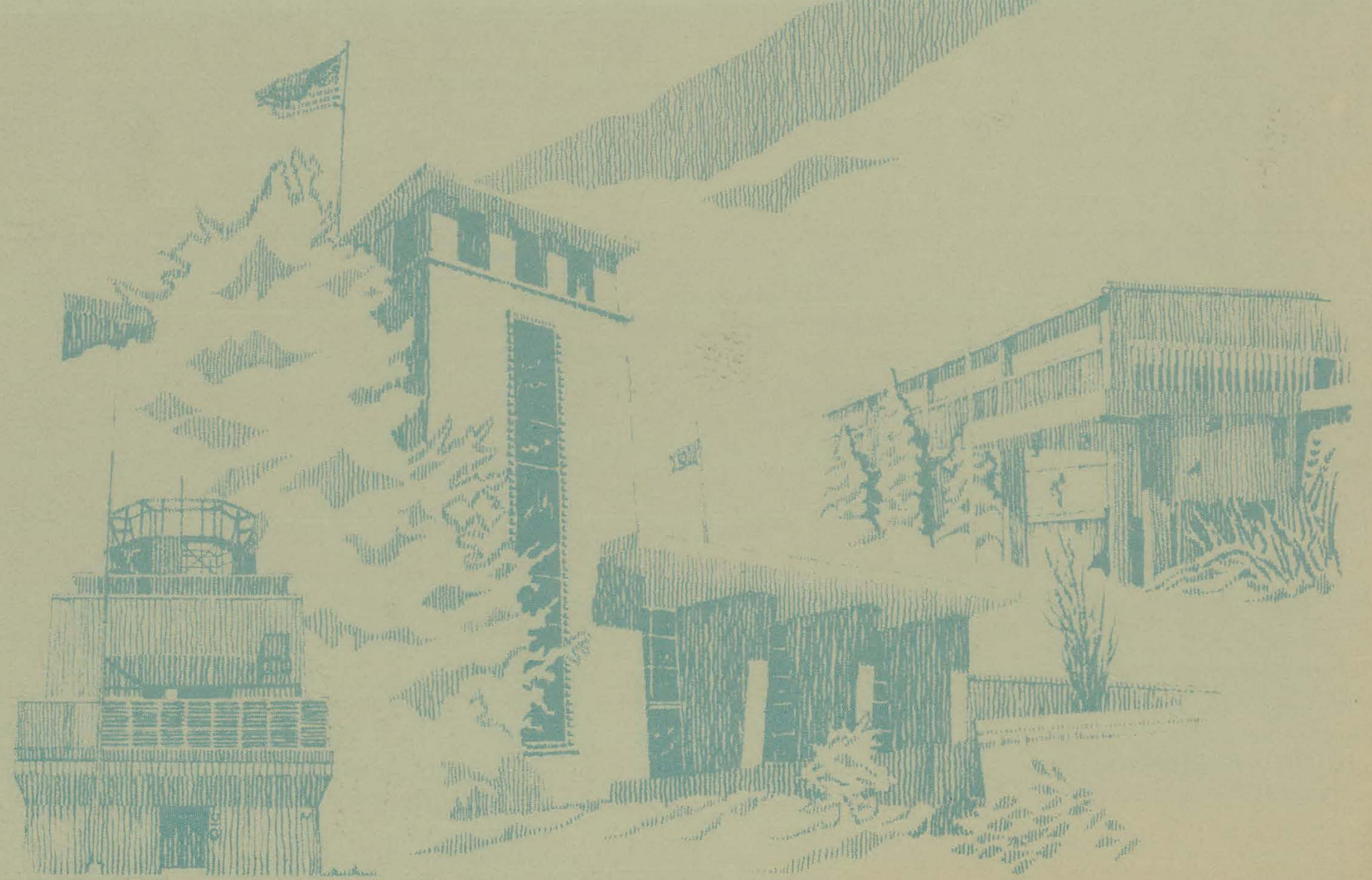




\section{DISCLAIMER}

This report was prepared as an account of work sponsored by an agency of the United States Government. Neither the United States Government nor any agency Thereof, nor any of their employees, makes any warranty, express or implied, or assumes any legal liability or responsibility for the accuracy, completeness, or usefulness of any information, apparatus, product, or process disclosed, or represents that its use would not infringe privately owned rights. Reference herein to any specific commercial product, process, or service by trade name, trademark, manufacturer, or otherwise does not necessarily constitute or imply its endorsement, recommendation, or favoring by the United States Government or any agency thereof. The views and opinions of authors expressed herein do not necessarily state or reflect those of the United States Government or any agency thereof. 


\section{DISCLAIMER}

Portions of this document may be illegible in electronic image products. Images are produced from the best available original document. 
Issued by Sandia National Laboratories, operated for the United States Department of Energy by Sandia Corporation.

NOTICE: This report was prepared as an account of work sponsored by an agency of the United States Government. Neither the United States Government nor any agency thereof, nor any of their employees, nor any of their contractors, subcontractors, or their employees, makes any warranty, express or implied, or assumes any legal liability or responsibility for the accuracy, completeness, or usefulness of any information, apparatus, product, or procompleteness, or usefulness of any information, apparatus, product, or pro-
cess disclosed, or represents that its use would not infringe privately owned rights. Reference herein to any specific commercial product, process, or service by trade name, trademark, manufacturer, or otherwise, does not necessarily constitute or imply its endorsement, recommendation, or favoring by the United States Government, any agency thereof or any of their contractors or subcontractors. The views and opinions expressed herein do not necessarily state or reflect those of the United States Government, any agency thereof or any of their contractors or subcontractors.

Printed in the United States of America Available from

National Technical Information Service

U.S. Department of Commerce

5285 Port Royal Road

Springfield, VA 22161

NTIS price codes

Printed copy: $\$ 6.00$

Microfiche copy: A01 
SAND 81-2476

Unlimited Release

Printed November 1981

SAND $--81-2476$

DE82 006878

REMOTE JOB ENTRY (RJE) FOR VAX/VISS
USER GUIDE

N. H. Samuelson

C. D. Brown

Division 2644

Sandia National Laboratories

Albuquergue, New Mexico 87185

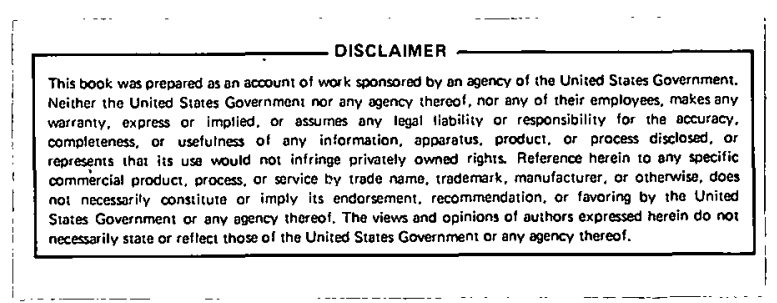

ABSTRACT

At Sandia National Laboratories in Albuquerque, New Niexico, a group of CDC computers handle large computational (ivatch) jobs. Some DEC VAx systems have been connected to the CDC systems to provide pre- and postprocessing of batch jobs. To facilitate running jobs on the CDC systems, members of Division 2644 created a Remote Job Entry (RJE) system. This document describes procedures for using that system. 
1. What is RJE? 4

2. How is RJE Accessed on the VAX? 5

2.1 Running the RJEUSER Program From Your Terminal 5

2.2 Command Language Interface to RJE 5

2.3 Output From RJEMGR

3. RJE Commands 6

3.1 Inquiry Commands $\quad 6$

3.1.1 STATUS 7

3.1 .2 STATUS, INPUT [INPUT]

3.1 .3 STATUS, LOCAL [LOCAL] 7

3.1.4 STATUS, OUTPUT [OUTPUT] , 7

3.1 .5 STATUS, QUEUE [QUEUE]

3.1 .6 STATUS, SITE [SITE] 7

3.2 Job Submittal, Control, and Retrieval 7

3.2.1 CANCEL 8

3.2.2 CLASSIFY 8

3.2.3 DIVERT -

3.2 .4 EVICT

3.2 .5 GET. 8

3.2.5.1 Qualifiers 10

3.2 .6 KILL 10

3.2 .7 NEXT 11

3.2.8 SENDJOB [EUBMIT] . . . 11

3.2.8.1 Passwords 11

3.2.8.2 Job Qualifiers 12

3.2.8.3. File Qualifiers 13

3.2.8.4 Defaults 13

3.3 Operator Privileged Commands 14

3.3.1 AUTO $\quad 15$

3.3.2 CONTINUE

3.3.3 LIMITS 15

3.3 .4 ON 15

3.3 .5 OFF 16

3.3 .6 PAIJSE. 16

3.3.7 SHUTDOWN 16

3.4 Miscellaneous Commands 16

3.4.1. MESSAGE 16 


\section{CONTENTS--Continued}

$\underline{\text { Page }}$

4. Text File Formats 17

4.1 Text Input Files 17

4.1.1 The "Control Statement" File 17

4.1.2 Other Text Input Files 18

$\begin{array}{lll}4.2 & \text { List Output } & 18\end{array}$

5. Binary File Formats 19

5.1 Binary Output $\quad 19$

5.1.1 Binary Conversion Routines 19

5.2 Binary Input Files $\quad 19$

5.2.1 Binary Conversion Routines 20

6. CDC Control statements of Special Interest to VAX Users 20

6.1 DISPOSE $\quad 20^{\circ}$

6.2 ROUTE 21

7. Examples 21

7.1 Minimal Job 21

7.2 COMPILE and RUN . . 22

7.3. Job with Binary Output 23

$\begin{array}{lll}7.4 & \text { Job with Binary Input } & 25\end{array}$

7.5 Routing Output to Another RJE Station 26

$\begin{array}{lll}7.6 & \text { Unsolicited Output } & 27\end{array}$

7.7 Shipping a Text File From One VAX to Another 28 


\section{What is RJE?}

The RJE subsystem runs on a VAX computer connected to the CDC batch computers through a ModComp front-end. It is similar in concept and operation to the traditional RJE stations that also connect to the $C D C$ batch systems through a similar ModComp front-end. A user logged into the VAX system can use the RJE system to do any of the following.

- Submit a job to run on the CDC systems and receive output from that job.

- Transfer either binary or text files in either direction between the VAX and the CDC systems (by submitting a job).

- Query the status of the lucal RJE system.

- Query the status of the CDC systems.

- Control jobs submitted to the CDC systeins (EVICT, DIVERT).

- Receive output from the CDC systems, even if the job was not submitted from that VAX. (It is possible with this mechanism. to transfer files from one VAX to another, $U$ l Lu sulnit a job from a VAX and get output on a traditional RJE station.)

Because the CDC batch systems run some classified work, only those VAXs that do NOT have dialup access can be connected to the batch systell by means of RJE. Submission of classified jobs, or retrieval of classified output, through RJE is not allowed at this time.

The RJE system on the VAX consists of three cooperating programs:

RJEMGR

The manager of the RJE system. It maintains the queues and manages operation of the RJE system. Normally it starts when the VAX system is loaded and should run continuously, waiting for requests to service.

RJEPUMP Communicates with the CDC systell. It is completcly controlled by the RJEMGR, and should not concern users.

RJEUSER The user interface. This program is run by any user who wishes to use the RJE package in any way. From the user's perspective, this is the RJE package. 
2. How is RJE Accessed on the VAX?

There are two ways to access the RJE system on the VAX: run the RJEUSER program and give it commands; or define new commands to DCL (the Digital command Language) that run RJEUSER in a transparent way.

2.1 Running the RJEUSER Program From Your Terminal

When the RJE system is installed on a VAX system, the command "RJE" is defined to allow a simple way to run the RJE user interface. The user interface is a privileged system program that parses commands from the user, formats requests to the manager of the RJE system, and displays output from the RJE system on the user's terminal.

When you give the command

$\$$ RJE

the RJEUSER program is run and types the prompt

RJE>

on your terminal. Now you can give any of the commands described in this report. After execution of any of those commands (except EXIT), or after a command error, RJEUSER returns to the RJE> prompt.

\subsection{Command Language Interface to RJE}

It is possible to define commands to DCL that will run the RJE user interface to execute single RJE commands in a transparent way. After execution of the RJE command, or after a command error, RJEUSER exits and returns to DCL. For example, if the following commands are defined to DCL like this:

$$
\begin{aligned}
& \$ \text { STAT }:==\text { RJE STATUS } \\
& \$ \text { WH }:==\text { RJE WHERE }
\end{aligned}
$$

then you can give commands like

$$
\begin{array}{ll}
\$ \text { STAT } & \text { :equivalent to RJE> STATUS } \\
\$ \text { STAT I } & \text { :equivalent to RJE> STATUS I } \\
\$ \text { WH XYZ } & \text { :equivalent to RJE> WHERE XYZ }
\end{array}
$$

In the rest of this report, all commands are described as they are given directly to RJEUSER, NOT through this DCL interface. 


\subsection{Output From RJEMGR}

As long as a user is running RJEUSER, RJEMGR can send messages to a user at his terminal notifying him of significant events such as the return of a job's output from the CDC systems. However, if the user is not running RJEUSER or has logged off the VAX and RJEMGR has a message to send to the user, then RJEMGR uses the VAX MAIL facility. The mail message may indicate that it came from "SYSTEM" or "RJE" (or some other privileged user) depending on huw RJEMGR was last started. In any case, the subject of the mail message will always say that it is a message from the RJE manager.

\section{3. $\overline{R J E}$ Commands}

All commands to RJEUSER are described in this section. They are grouped ae

- Inquiry commands

- Job submittal, control, and retrieval commands

- Operator privileged commands

- Miscellaneous commands

All commands can be abbreviated to the shortest unique substring. For example, WHERE can be abbreviated WHER, WHE, WH, or just $W$. Some commands, for the sake of compatibility, also have other abbreviations or synonyms. Synonyms are shown in brackets after the command name in the following sections.

\subsection{Inquiry commands}

The commands in this group get information from RJEMGR and/or from the CDC system (BART). Messages from RJEMGR in the VAX are in mixed case and messages from the CDC BART package are in all upper case. Most commands are identical to commands used on the non-VAX RJE stations. The only exceptions to this rule are the following.

- The command $L$ is used for STATUS, LOCAL rather than LIMITS.

- Some commands do not make sense on the VAx. An example is REWIND, which restarts the job on the local printer on non-VAX RJE stations. 


\section{1 .1 STATUS}

The STATUS command with no arguments is equivalent to the STATUS, QUEUE command.

3.1 .2 STATUS, INPUT [INPUT]

The STATUS, INPUT command returns information from BOTH RJEMGR and CDC. The local status shows the contents of the local VAX input queue; the CDC status shows the contents of CDC input queues.

3.1.3 STATUS, LOCAL [LOCAL]

The STATUS,LOCAL command returns the number of entries in each of the local queues, and lists each of the entries that belongs to you. It does not query the $C D C$ system, and returns no information about it other than an informative message if the link is down.

\subsubsection{STATUS, OUTPUT [OUTPUT]}

The STATUS, OUTPUT command returns information from BOTH RJEMGR and CDC. The local status shows the contents of the local output queue; the CDC status shows the contents of the CDC output queues.

\section{1 .5 STATUS, QUEUE [QUEUE]}

The STATUS, QUEUE command returns information from $C D C$ about the number of jobs in the CDC input and output queues.

\subsubsection{STATUS,SITE [SITE]}

The STATUS,SITE command returns information about the status of the local site from CDC--which data streams are up, what are the limits, whether a data transfer is active, etc.

\subsection{JOB SUBMITTAL, CONTROL, and RETRIEVAL}

Before discussing each command in detail, we define a few useful gencral terms:

Equipment (data stream). The data streams are pseudodevices that correspond to simultaneous file transfers across the communications link. Those supported by, the VAX RJE package are

CR "Card Reader" stream, input to CDC 
LP "Line Printer" stream, list output from CDC

PCH "Punch" stream, binary output from CDC

LFN

Logical File Name. All jobs in the CDC system are identified by a seven-character LFN. Entries in some of the RJE queues are also identified by this name. The name is created by the CDC system when a job is submitted. The first four characters come from the "JOB CARD", the next two identify which RJE station submitted the job, and the last two are assigned in sequence by the CDC system to limit the possibility of duplicate job names.

The full seven characters must be used in most RJE commands requiring an LFN. Exceptions include WHERE and CANCEL. When a short form is allowed, at least three characters must be given, and any job that matchee the characters specified is treated as. a match; i.e.: WHERE, XYZ finds all jobs whose LFN starts with $X Y Z$.

Unsolicited Output

Output sent from the CDC systems to a VAX for which no corresponding entry exists in the local output queue.

\subsubsection{CANCEL}

This command removes an entry from one of the local VAX queues maintained by RJEMGR. If a jub is cancelled while still in the VAX input queue, i.e.. it has never been sent to the CDC systems, then it is discarded. That is the end of it. If a job is in the VAX output queue when cancelled, the VAX output queue entry is discarded and any output that comes back for that job is treated as unsolicited output. The format of the command is

\section{CANCEL, Ifn}

where the LFN is the temporary job ID assigned by RJEMGR if the job is still in the input queue, or the CDC job ID if the job has already been sent to the CDC system. If you specify less than seven characters, ALL jobs belonging to you whose first characters match the part you specified are cancelled. For example, if you always use XYZ as the job name, you can cancel ALL your jobs with the command: 


\subsubsection{CLASSIFY}

This command is NOT yet allowed. It will be used in the future to retrieve classified output after the VAX/VMS systems are enhanced to handle classified data.

\subsubsection{DIVERT}

Divert a job in one of the $C D C$ output queves to the central site printer or card punch. The format of the command is

$$
\text { DIVERT, lfn, } q
$$

where $\mathrm{q}$ is either OUTPUT or PUNCH

\subsubsection{EVICT}

Remove a file from one of the CDC queues. The format of the command is

$$
\text { EVICT, } 1 \mathrm{fn}, \mathrm{q}
$$

where $Q$ is the name of the CDC queve; i.e.:

$$
\begin{array}{ll}
6 & \text { Input queue for the } 6600 \text { systems } \\
7 & \text { Input queue for the } 7600 \text { systems } \\
\text { OUTPUT } & \text { Output queue from either system } \\
\text { PUNCH } & \text { Binary output queue from either system }
\end{array}
$$

A job in the VAX RJE local OU'lyuT queue may be in the $C D C$ INPUT queue, and it is the $C D C$ queue name you specify in the EVICT command. Because EVICT also implies CANCEL, the job is removed from the local VAX queues as well as from the CDC queues.

\section{2 .5 GET}

The GET command has two similar uses. If unsolicited output has been or will be sent to the VAX RJE system, the output cannot normally be routed to the correct user. The GET command retrieves files from the unsolicited output queue. If the output has not yet come from the CDC system, it places an entry into the output queue so that the 
output can be routed correctly when it arrives. The form of the GET command is

GET Ifn (/qualifiers)

3.2.5.1 Qualifiers

/LIST=file-name

Specifies where the LIST output files are to be delivered. The default is your current default disk and directory, with the LFN as the file name and. LOC as the file type (extension).

$/ P U N C H=f i l e-n a m e$ Specifies where the PUNCH output files (if any) are to be lelivered. If the file name is omitted, the default is your current default disk and dirprtury, with Llut LF! as the file name and. BIN as the file type (extension).

/CARRIACE CONTROL- Spegifies the type of rarriage control to be usea wllen lite file is returned from the CDC system. Two pussible keywordg are used here:

FORTRAN The first column of output is carriage control (this is the default).

LIST The first column of output is part of.the text.

3.2 .6 KILL

Kill the currently active output file on the indicated device. The format. is

KILL, eq, Ifn

wherc EQ is one of LP, PCH, or CR. 


\subsubsection{NEXT}

The NEXT command raises the priority of a file returned to the $C D C$ system (perhaps because the user exceeded his disk quota on the VAX) and causes that file to be retransmitted to the VAX. If a user gets a message from RJEMGR saying a job was returned to the CDC system, he should make enough room for the file and then use the NEXT command to retrieve it. The format is

$$
\text { NEXT, eq, lf }
$$

where eq is either LP or PCH.

\subsubsection{SENDJOB [SUBMIT]}

Jobs submitted to the CDC batch systems consist of one or more files that may be thought of as a "card deck." It is possible to control the structure of that "card deck" in a few ways. The normal mode of operation is to separate each input file with an "end of record," and to follow the last file with an "end of information." This can be altered by

- Qualifiers specified in the SENDJOB command.

- Special characters imbedded in the input files.

The qualifiers are described in this section; the special characters in the input file are covered in the section on Text File Formats.

The SENDJOB command requires one or more file names; e.g.

$$
\text { SENDJOB JI.CCL,JI.FOR, JIA.DAT }
$$

\subsubsection{Passwords}

With the current implementation of the CDC systems, every job must have at least one password, and may include many passwords (for file accesses). RJE provides a simple mechanism to eliminate the need for passwords in files on the VAX. See section 4.1.1 for details. 


\subsubsection{Job Qualitiers}

The following qualifiers are recognized anywhere in SENDJOB commands and apply to the job as a whole.

/LIST

/LIST=file-name

/NOLIGT

/PUNCH

/PUNCH $=$ file-name

/NOPUNCH

/TIME LIMIT $=\mathrm{n}$
Specifies where the LIST (text) output files are to he written when the output is returned from the CDC system. /OUTPUT is a sỸuvirym fü /LIST.

specifica that no liet output is expected. When the job is sent to the CDC system, the local queue cntry is delcted if no LIST or PUNCH output is expected. This is NOT the normal case.

Specifies that PUNCH (binary) output is experted, and is written to the file specified. The file name and "=" can be

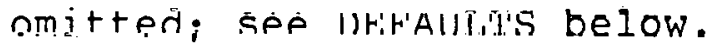

Specifies that no PUNCH output is expected. This is the default.

Specify the time limit (in DAYS) that the jub should stay in the output queue waiting for expected output. Entries in the output queue are deleted when all output has arrived, or when this time limit has expired. The default is 3 days. 
3.2.8.3 File Qualifiers

File qualifiers follow the corresponding file name.

/ASCII (D)

/BINARY

/SEGMENTED_BINARY Specifies the form of the input or output file. All input files are assumed to be ASCII text files unless one of the forms of binary is specified. All list output MUST be ASCII. Punch output files are assumed BINARY unless /SEGMENTED_BINARY is specified. NOTE: Normal VAX binary files will NOT get to the CDC system intact. See the section on Binary File Formats for details.

/EOF

$/ \operatorname{EOR}$ (D)

/CONCATENATE
[/EOP or /679]

[/EOS or /789]

[/NOEOR OR /NOEOF]

specifies that this file is to be separated from the following file (if any) by an "End of File", "End of Record", or no separator at all. The default between files is EOR, and the last file is ALWAYS followed by "End of Information".

/CARRIAGE_CONTROL=FORTRAN (D)

/CARRTAGE-CONTROT.t=LTST

Specify whether column 1 of. ALL text output files for this job is to be treated as carriage control. Because the default is FORTRAN, most files from the CDC will look correct if typed or printed on the VAX. If the first column of each line in the output file contains data, and not carriage control information, LIST should be specified.

\subsection{8,4 Defaults}

1. The default extension (file type) of the first file is ".CCL" (for Cyber Command Language). 
2. The default directory and device for the first file are your current default directory.

3. For files after the first, the default device and directory are the device and directory last specified in the command, or your current defaults if none has been specified (i.e., once a device or directory is specified it "sticks" through that command unless changed again), For example, if your current default is UD: [MYDIR], the command

SENDJOB J1,SD:P1.FOR, [XYZ]DI.DAT, UD:XX

looks for the following flles

UD: [MYDIR] J].CCL

SD: [MYDIR] PI.FOR

SD: $[X Y Z] D 1 . D A '$

UD: $[X Y Z] X X$.

4. For all files except the first, there is no default file type (extensinn).

5. Each file is, by default, separated from the next by an "end of record."

6. The LIST files default to your current default directory, with the same name as the .CCL file, and the file type (extension) of .LOG.

7. The PUNCH files default to your current default directory, with the same name as the .CCL file, and the file type (extension) of . BIN. This is true ONLY if you epecify /PUNCH in the SENDJOB command; otherwise, no punch output is expected.

\subsection{Operator Privileged Commands}

The commands contained in this section require OPER privilege not normally granted to most VAX users. They allow changes in the operation of the RJE system that can affect all users of the system.

BEWARE

Users with OPER privilege will see all jobs for ALL USERS when looking at the local queues maintained by RJEMGR, and can CANCEL a job for ANY 
user EVEN ACCIDENTALLY when using the short form of the CANCEL command.

\section{3 .1 AUTO}

Turn the AUTO DIVERT switch on or off in the CDC system. This command should NOT be used except in very unusual circumstances; $i . e .$, the AUTO DIVERT switch should normally stay OFF, so that all output is sent back to the VAX. The format of the command is

$$
\text { or AUTO, OFF }
$$

\subsubsection{CONTINUE}

The CONTINUE command resumes I/O suspended by a PAUSE command. The format of the command is

$$
\text { CONTINUE, eq }
$$

where $E Q$ is one of LP, PCH, or CR.

\section{3 .3 LIMITS}

The LIMITS command sets a limit on the size of output files that are sent to the VAX system. Any file larger than the upper limit (or smaller than the lower limit) is held in the output queue at the CDC system. The format of the command is

\section{LIMITS, eq, upper, lower}

where $E Q$ is either $L P$ or $\mathrm{PCH}$. If either limit is omitted, it means "leave it as it is." If the upper limit is omitted, two consecutive commas are required (i.e.: LIMITS,LP, 0 ). The LOWER limit should ALWAYS be zero. Note: On non-VAX RJE stations, the command $L$ is an abbreviation for LIMITS. On VAX RJE systems, $L$ is an abbreviation for the STATUS, LOCAL Commalid.

\section{3 .4 ON}

Turn on a data stream (logical device) for input to, or output from, the CDC system. The format of the command is

$$
\text { on, eq }
$$

where EQ is one of LP, PCH, or CR. 


\section{3 .5 OFF}

Turn off a data stream (logical device) for input to, or output from, the CDC system. The format of the command is

$$
O F F \text {, eq }
$$

where $E Q$ is one of $L P, P C H$, or $C R$. The stream is shut down AFTER any currently active file transfer is complete.

\subsubsection{PAUSE}

Suspend $I / O$ on the specified data stream (logical device). The format of the command is

$$
\text { PAUSE, eq }
$$

where $\mathrm{EQ}$ is onc of LP, PCH, nr CR. The CONTINUE command is the inverse of PAUSE.

\subsubsection{SHUTDOWN}

Reyuesl that RJEMGR chut itself down. if snme data stream is currently active, it will continue; but no new file transfors will be started. If the stream is still active 5 minutes later, RJEMGR assumes it was hung and shuts itself down anyway.

\subsection{Miscellaneous Commands}

\subsubsection{MESSAGE}

The MESSAGE command sends a text string to the CDC operator. The format of the command is

$$
\text { MESSAGE any string of text }
$$

Example:

\section{MESSAGE HELP, THIS VAX IS ON FIRE}

Note: This command should NOT be used in normal circumstances. If the CDC operator responds; the VAX RJE system doesn't know where to route the reply and thus sends it to EVERY TERMINAL on the VAX. This feature is meant to handle special messages only, such as telling all VAX users that the CDC systems are on fire. 
4. Text File Formats

\subsection{Text Input Files}

Text files are normal VAx text files. The character set on the VAX is ASCII. Files are converted to DISPLAY CODE in the ModComp front-end while going from the VAX to the CDC system, and from DISPLAY CODE to ASCII while going from the CDC system to the VAX. Because DISPLAY CODE is a 64-character set, all lower-case characters are converted to upper case going in to the CDC system. Tabs are expanded to some number of spaces between 1 and 8 , to get to the next "tab stop." Tab stops are fixed at columns 1 , 9. 17, etc.

When the files are sent to the CDC system, they are scanned for imbedded separator indicators, such as " $\{$ EOR $\}$ " or " $\{$ EOF\}". These indicators MUST start in column 1. When such an indicator is found, an end-of-record or end-of-file is sent to the CDC system.

\subsubsection{The "Control statement" File}

The first file in a job is treated as the control statement file. The second control statement in a CDC job should be a USER statement that contains a user identifier and password. CDC file access commands containing passwords may also appear in the control statement file. Because it is undesirable to have CDC passwords appearing in VAX files, a password replacement feature is provided in RJEUSER. When an RJE SENDJOB command is entered, RJEUSER copies the control statement file to a "hidden" file on the VAX. Each time a text string in braces is encountered, the user is prompted with the text string. Whatever text he enters is substituted in the "hidden" file for the original text string including the braces. For example, the CDC control statement

$$
\text { USER, JDSMITH, \{NOS PasSword\}. }
$$

would result in the prompt

\section{Enter "NOS Password":}

When the user enters his password it will be substituted in the USER statement. The password will not be echoed so that other users cannot observe it. 
If a user wishes to ensure that the password is entered correctly, he may place an exclamation point at the end of the prompt string just before the closing brace. In that case RJEUSER will prompt twice for the password and will verify that it is entered the same way both times. If it is not, the SENDJOB command will be immediately aborted. This verification mechanism may be useful in cases where a mistake could result in the loss of several hours of CDC time, e.g., when an output file from a lengthy run is being saved in the permanent files.

\section{WARNING}

Password replacement is not done on strings whon the opening brace is in Column 1 of a line, since $\{$ EUK $\}$ and $\{\mathrm{EOF}\}$ must start in Column 1 .

since the control statement tile is coplen to a "hidden" file, it is not wise to make it a very large file with a lot of data following the control statements. The time reyuiled fui RJEUSer to copy a large file may be noticeable, and a considerable amount of the user's disk quota may be consumed às a result.

This string replacement mechanism is NOT restricted to passwords only, but can be used as a general parameter replacement mechanism.

\subsubsection{Other Text Input Files}

The other input files are NOT copied when you give the SENDJOB command. They must not be deleted before the job is actually sent to the CDC system. They will be scanned for imbedded separators such as " $\{E O R\} "$ or " $\{E O F\} "$, but password replacement will NOT be done.

\subsection{List output}

All text output directed to the file "OUTPUT" on the CDC system is delivered to the list output file specified explicitly or implicitly in the SENDJOB command. If the OUTPUT file contains imbedded end-of-file marks, it is delivered as multiple "versions" on the VAx; i.e., files with the same name and extension, but different version numbers. For example, a compilation listing followed by program output and by the dayfile comes back as three versions. 
5. Binary File Formats

5.1 Binary output

Note: Unless you specify/PUNCH in your SENDJOB command, the VAX does not expect binary output from your CDC job and will not know how to deliver it to you. See the section on Unsolicited Output for more information on what happens to it. Each 60-bit CDC word is stored on the VAX in 8 consecutive bytes $(64$ bits), with the upper 4 bits of the first byte set to zero. If the file contains imbedied end-of-file marks, it is delivered as multiple "versions" on the VAX; i.e., files with the same name and extension, but different version numbers.

The qualifiers/BINARY and /SEGMENTED BINARY refer to the method in which binary data is written on the VAX. The RJEPUMP process writes normal BINARY output in variable length records of up to 4096 bytes each. CDC records longer than 4096 bytes are broken into multiple 4096 byte records with a short record at the end.

SEGMENTED BINARY records are written as a sequence of short variable length records of 126 bytes or less, of which the first two data bytes are actually flag bytes that indicate whether that record is the beginning, middle, last, or only record in the larger "segmented record." segmented records are a special feature of VAX-1l FORTRAN (they are the default type for unformatted reads and writes) and are not recognized by the record manager as a separate record type. Instead, RMS just treats a segmented record as a string of short variable length records. Segmented binary records are of particular use when CDC record sizes exceed 4096 bytes ( 512 CDC words), since the VAX segmented records will have a one-to-one correspondence with CDC recoras.

5.1.1 Binary Conversion Routines

Routines are available to convert the binary format used for output from the $C D C$ system to normal VAX binary formats. Those routines are included in the library CDCBIN.OLB and are documented in the file CDCBINARY.MEM, which should be available on each VAX system with an RJE connection.

\subsection{Binary Input Files}

It is NOT possible to send just any VAX binary file to the CDC system. The file MUST be in a format like that used for binary output from the CDC system, in which the first 
4 bits of every 64 bits must be zero. They are stripped off to fit into the 60-bit word of the CDC system. If the contents of 8 consecutive bytes look like

$O A \quad B C$ DE FG HI JK LM NP

where each letter represents a 4-bit hexadecimal digit, then it gets to the CDC system looking like

ABC DEF GHI JKL MNP

\subsubsection{Binary Conversion Routines}

Routines are available to convert normal VAx binary formats to the proper format to be sent to the CDC system. Those routines are included in the library CDCBIN.OLB and are documented in the file CDCBINARY.MEM, which should be available on each VAX system with an RJE connection.

\section{CDC Control statements of special Interest to VAX users}

While we do not want to go into any unnecessary details about general CDC systems, there are a few control statements of particular interest to VAX RJE users.

\subsection{DISPOSE}

The DISPOSE statement disposes a file either immediately or at the end of the job. Because of the way the queues are maintained on the VAX, we S'IRUNGLY recommend inclusion of the $*$ in the DISPOSE statement so that the output will ALL come at the end of the job if output is to be sent back to the VAX from which the job was submitted. To dispose a file to OUTPUT:

DISPOSE, $1 f n, \star \bar{P} R$. or DISPOSE, If $n, P R, S T=N O S E X X$.

Where $\mathbf{x} x$ is the RJE station (example: NOSER2 or NOSEV3). To dispose a binary file to the VAX (as punch output): DISPOSE, lfn, $\star \mathrm{P} 8=\mathrm{CVX}$. or DISPOSE, LFN, P8=CVX, ST=NOSEXX. 
6.2 ROUTE

The ROUTE command can be used on OPEN NOS to route a file to a VAX connected to the SECURE batch systems. The form of the ROUTE command is

ROUTE, If , DC=SO, TID $=x \times$.

7. Examples

In all the examples in this section, we assume the user is John Q. Sandia, and that Mister Sandia is known to NOS as JQSANDI, has password MYPASWD, and likes to use his initials for his CDC job name. All commands that he types on his terminal are underlined in all examples; all other text constitutes either system prompts or comments.

\subsection{Minimal Job}

First, consider the minimum useful job. List the contents of your directory on Nos. Only one file will be needed; let's call it MINI.CCL. The contents of that file would look like

JQS .

USER, JQSANDI, \{NOS Password\}.

$\mathrm{CNH}$.

Mr. Sandia would type the following commands on his VAX terminal:

\section{$\$$ RJE}

RJE SEND MINI

Enter "NOS Password": MYPASIND

[Sending Job JQS37]

RJE>

JQSOEAN PLACED INTO QUEUE

Temporary job name was JQS37, 32 bytes transmitted

RJE>

When the job finishes execution on the CDC system, Mr. Sandia is notified at his terminal (if he is still logged in).

978 bytes of List output received for Job JQSOEAN Job Completed

RJE > EXIT

Mr. Sandia now finds a file named MINI.LOG in his directory. It contains the dayfile and directory listing from his job. 
7.2 Compile and Run

The next example includes compilation and execution of a FORTRAN program on the 7600. There are two options for this:

- The control statements are in one file and the program in another file.

- The control statements and program are in the same file, separated by one line containing only " $\{E O R\}$ ".

For this example they are combined in a single file; in following examples they are shown as separate files. The contents of the file "EXAMPLE2.CCL" are

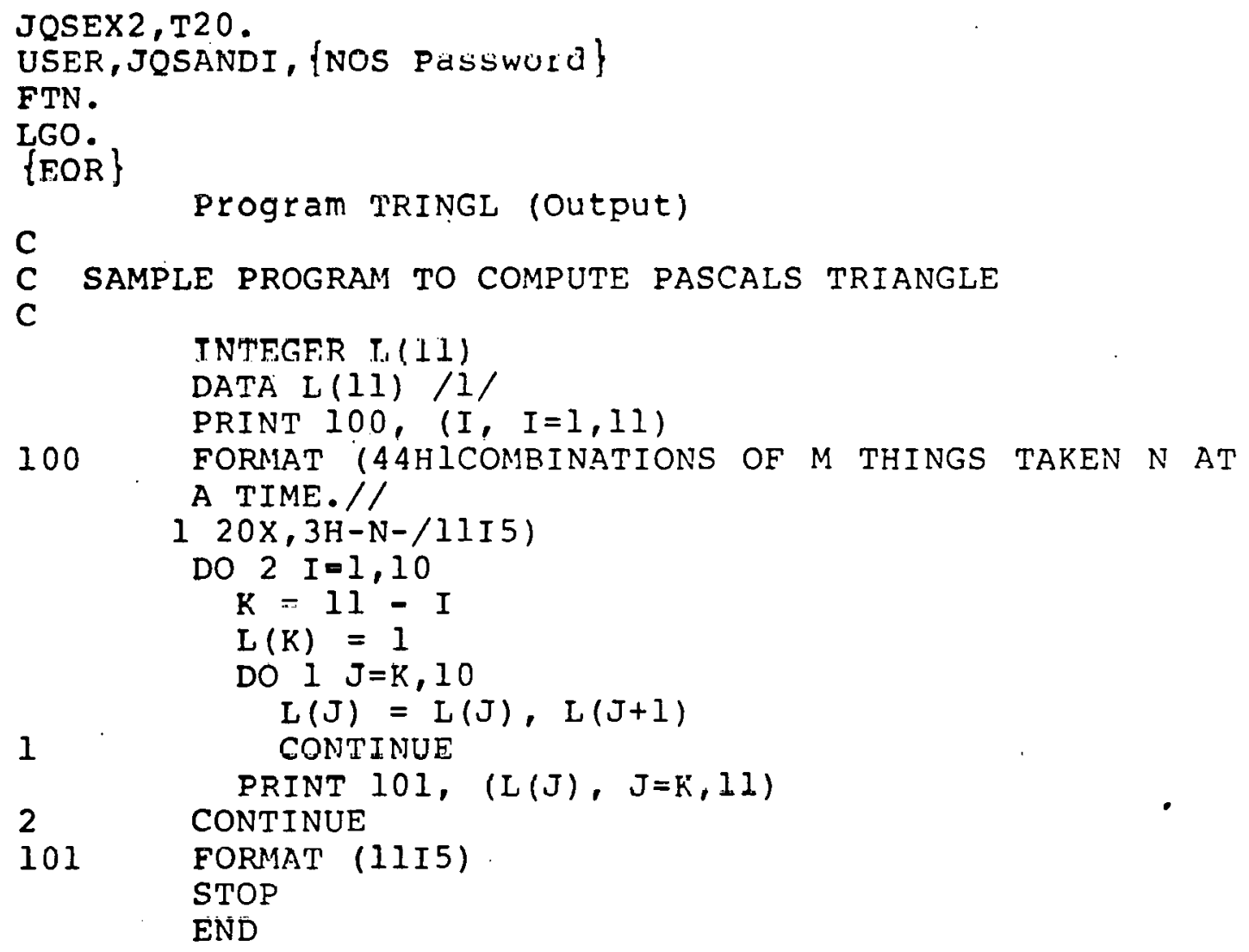

To send this job to the CDC system, Mr. Sandia types the following commands on his VAX terminal:

$\$$ RJE

RJE'S SEND EXAMPLEZ

Enter "NOS Password": MYPASWD

[Sending Job JQS83] 


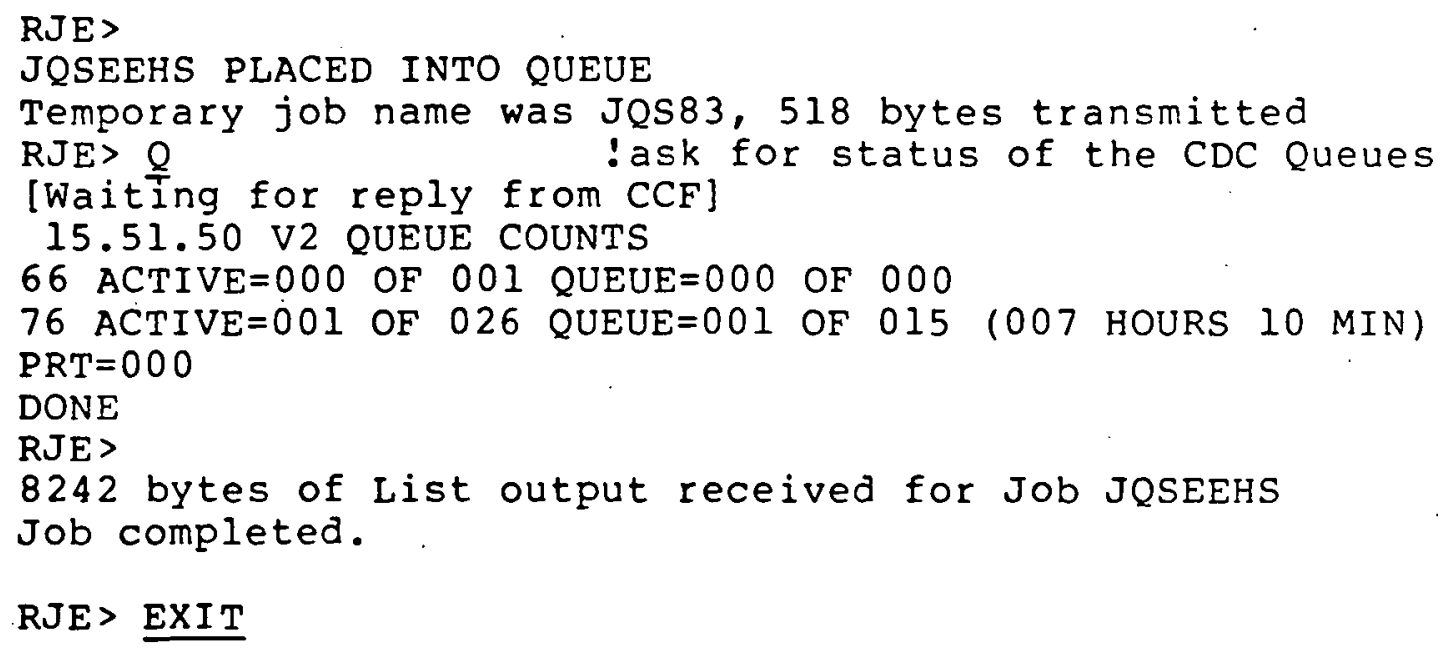

Mr. Sandia will now find Two files named EXAMPLE2.LOG in his directory. The file with the highest version number is the dayfile; the lower numbered version is the output of his program.

\subsection{Job with Binary Output}

In this example Mr. Sandia runs a program that creates a binary output file on the $C D C$ system and returns that file to the VAX. The file EXAMP3.CCL contains the following:

JQS (T10)

USER, JQSANDI, \{PSWD $\}$.

FTN5.

LGO.

DISPOSE, TAPEI, P8 $=$ CVX.

The file EXBIN.PGM looks like

PROGRAM FLOAT (OUTPUT, TAPEI)

REAL $X(1000)$

DATA $(X(I), I=1,10) / 1.0,0.0,3.3333333,100 \ldots, 1.89 E+150$,

$1-1.0,-0.0,5.5 \mathrm{E}-40,-2.666666667 \mathrm{E}+9,-6.5891432 \mathrm{E}-4 /$

DO $10 \quad I=1,991,10$

$$
\begin{aligned}
& \text { DO } 10 \mathrm{~J}=\mathrm{I}, \mathrm{I}+9 \\
& X(J)=X(J-I+I)
\end{aligned}
$$

10 CONTTNIIF.

PRINT 100, (X(J), J=1,100)

100 FORMAT $(1 \mathrm{X}, 216,4 \mathrm{X}, \mathrm{216})$

DO $20 \quad I=1,10$

$$
\text { WRITE (I) (X(J), J=1,1000) }
$$


20

CONTINUE

STOP

END

To send this job to the CDC system, Mr. Sandia types the following commands on his VAX terminal:

$\$$ RJE

RJE> SEND EXAMP3,EXRIN.PGM/PUNCH

Enter "PSWR": MYPASWR

[Sending Job JWS84]

RJE>

JQSOEJO PLACED INTO QUEUE

Temporary job name was JQS84, 491 bytes transmitted

RJE? $Q$

[Waiting for reply from CCF]

15.51.50. V2 QUEUE COUNTS

66 ACTIVE $=000$ OF 001 QUEUE $=000$ OF 000

76 ACTIVE $=000$ OF 033 QUEUE=002 OF (007 HOURS 55 MIN)

PRT $=000$

DONE

RJE> EXIT

$\$ \underline{\text { LOGOUT }}$

In this case, Mr. Sandia decides not to wait at his terminal until the job is run. He sees the following the next time he logs in to the VAx:

You have 2 new messages

When he runs MAIL to read them, he sees the following:

$\$ \operatorname{ma11}$

you have 2 new messages

MAIL> read

From: KJE 12-AUG-1981 14:04

TO: JQSANDI

Subj: Message from RJE system:

80000 bytes of Punch output received for Job JQSOEJO

More output expected.

MAIL > de 1

MAIL $>\underline{\text { read }}$ 
From: RJE 12-AUG-1981 14:05

TO: JQSANDI

Subj: Message from RJE system:

12180 bytes of list output received for Job JQSOEJO Job completed.

MAIL> de 1

MAIL> $\overline{\text { read }}$

8MAIL- $\overline{E-N O M O R E M S G, ~ n o ~ m o r e ~ m e s s a g e s ~}$

MAIL> exit

Mr. Sandia now finds TWO files named EXAMP3.LOG and ONE file named EXAMP3. BIN in his directory. The .BIN file is the binary output (i.e.: TAPEl); the . LOG files are the dayfile and the output from his program, as in Example 2.

Because the floating-point numbers in the binary output file are still in the CDC internal representation, they can't be used yet. They MUST be converted to VAX internal floating-point format before they can be used to do any arithmetic on the VAX. Conversion would also be necessary for a CDC binary file containing integers or display code characters. See the chapter on Binary File Formats for more information.

7. 4 Job with Binary Input

In this example Mr. Sandia wants to send a binary file to the CDC system to be plotted at an adjacent old-style RJE station. The command file is named COMO.CCL and contains the following:

JQS, STNOS. COMQ SUBMITTED FROM VAX. USER, JQSANDI, \{Secure NOS Password\}. COPYBF, INPUT, TAPE 77 . COMQ, TAPE77, HCl, CS=R2 .

The binary file is called FORO78.DAT and contains data ALREADY FORMATTED CORRECTLY FOR THE CDC SYSTEM (see the section on Binary File Formats for details).

To send this job to the CDC system, Mr. Sandia types the following commands on his VAX terminal:

$\$$ sendjob comg, for 078. dat/binary

Enter "Secure NOS Password": MYPASID

[Sending Job JQS85]

In this case Mr. Sandia would get two mail messages from RJEMGR as the job progresses through the systems. 
The first message is sent when the files are transferred to the CDC systems. It gives the job name (LFN) assigned by the $C D C$ system and the count of the number of bytes transferred.

The second message comes after the job has been run and the dayfile returned to the VAX, saying that the job is complete and listing how many bytes of output were received.

7.5 Routing Output to Another RJE Station

In this example Mr. Sandia runs the same program as in Example 1, but routes the program output to the printer at an adjacent old-style RJE terminal.

The command file 1 s named PREMO'te.CCL and contains the following:

DEMO, T2U.

USER, JQSANDI, \{NOS PASSWORD\}.

FTN.

LGO.

DISPOSE, OUTPUT, PR, ST=NOSER2. Frint output at $R 2$

The program is in file TRINGL.FOR (see Example 1 for the contents of that program).

To send this job to the CDC system, Mr. Sandia types the following commands on his VAX terminal:

$\$$ RJE

RJ $\overline{E S S E N D J O B ~ P R E M O T E, ~ T R I N G L . F O R ~}$

Enter "NOS PASSWORD": MYPASWD

[Sending Job DEM86]

R.JE >

DEMOENN PLACED INTO QUEUE

Temporary job name was DEM86, 590 bytes transmitted

RJE $>0$

[Waiting for reply from CCF]

14.10.30. V2 QUEUE COUNTS

66 ACTIVE $=000$ OF 000 QUEUE $=000$ OF 000

76 ACTIVE $=000 \quad$ OF 032 QUEUE=001 OF $024 \quad 1009$ HOURS

10 MIN)

$\mathrm{PRT}=000$

DONE

RJE> 
2818 bytes of List output received for Job DEMOENN Job Completed.

\section{RJE> EXIT}

\subsection{Unsolicited Output}

This example does NOT include a sendjob command, because we are showing how a job that was NOT submitted from a VAX can get to a user on a VAX. For this example, Mr. Sandia logged into the OPEN NOS system and sent a file from there to VAX node V2 with the following command:

$$
\text { ROUTE, Ifn, DC=SO, TID }=V 2 \text {. }
$$

The CDC system responds with the job name (LFN) assigned to the job:

\section{ROUTE COMPLETE. JOB NAME IS JQSV2FH}

Some time later (after the file has been transferred from OPEN NOS to SECURE NOS by operations) the file arrives at VAX node V2. Mr. Sandia uses the GET command to request the output. If the file has already arrived, it is copied to his directory immediately. Otherwise, the GET command creates an entry in the local OUTPUT queue to await the arrival of the file. The LFN assigned by the CDC system must be used in the GET command. If a file name is specified. with the /LIST= switch, that name is used; otherwise the file name is the LFN with extension. LOG (or, for $/ \mathrm{PUNCH}=$, . BIN), i.e.:

$\$$ RJE

RJE $\overline{E D T}$ JQSV2FH/LIST=MYFILE.NOS/CA $=$ LIST

The response will be one of the following:

or

[Job placed in output queve]

List output will be copied to your directory

If the GET command is issued BEFORE the output is received, qualifiers such as /CARRIAGE_CONTROL=LIST or /SEGMENTED BINARY will have the desired effect. If the GET command $i \bar{s}$ issued AFTER the output has already been received, it is TOO LATE for those qualifiers to have effect. For this reason, text files should normally be copied on the CDC system with COPYSBF, rather than COPY, to insert FORTRAN-like carriage control in the first character position of each line. For SEGMENTED_BINARY files 
you must be FAST. Thus, writing shorter records that don't require segmentation is recommended.

\subsection{Shipping a Text File From One VAX to Another}

In this example, Mr. Sandia wants to send a program to a user on another VAX. The file we will send is the program used in the previous example; i.e., TRINGL.FOR.

In transferring text files by this means, characters are converted from ASCII to DISPLAY CODE, and later back to ASCII. This results in converting all lower case characters to uppercase, converting tabs to multiple spaces, and loss of some special characters.

The command file is named GIVEFILE.CCL and contains a destination node that can be varied by using the string replacement normally used for passwords, i.e.:

jgs(t2,stnos) job to move file to another VAx user,jusandi, \{Nos passwora\}.

copysbf, input, output.

route, output, $d c=p r, t i d=\{$ Destination node $\}$.

Mr. Sandia gives the following commands to send this job to node V3:

\section{$\$$ rje}

RJ $\overline{\mathrm{E}>S E N D}$ GIVEFILE, TRINGL.FOR

Enter "NOS password": MYPASWD

Enter "Destination node": V3

[SEliding Job JQSB7]

RJE>

JQSOEO 8 PLACED INTO QUEUE

Temporary job name was JQS87, 615 bytes transmitted

RJE $>\underline{E X}$

Mr. Sandia gets one more message when the job is completed, telling him that the dayfile has been received and including the byte count. The flle is sent to the nude he specified, wheie it is treated as UNSOLICITPR OITPIIT (unlose the user expectiny the rile issues a GET command DEFORE the file arrives). 
Distribution:

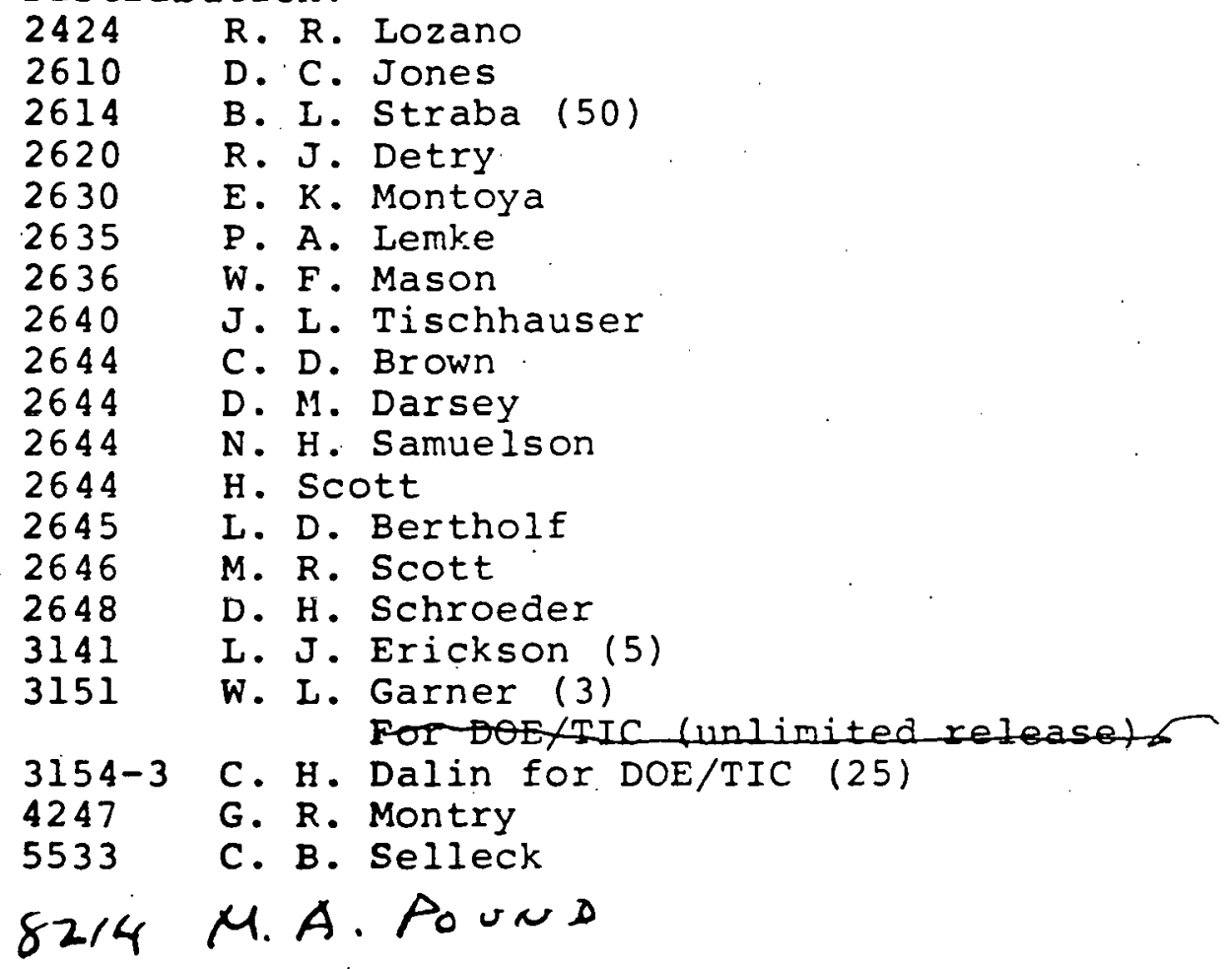




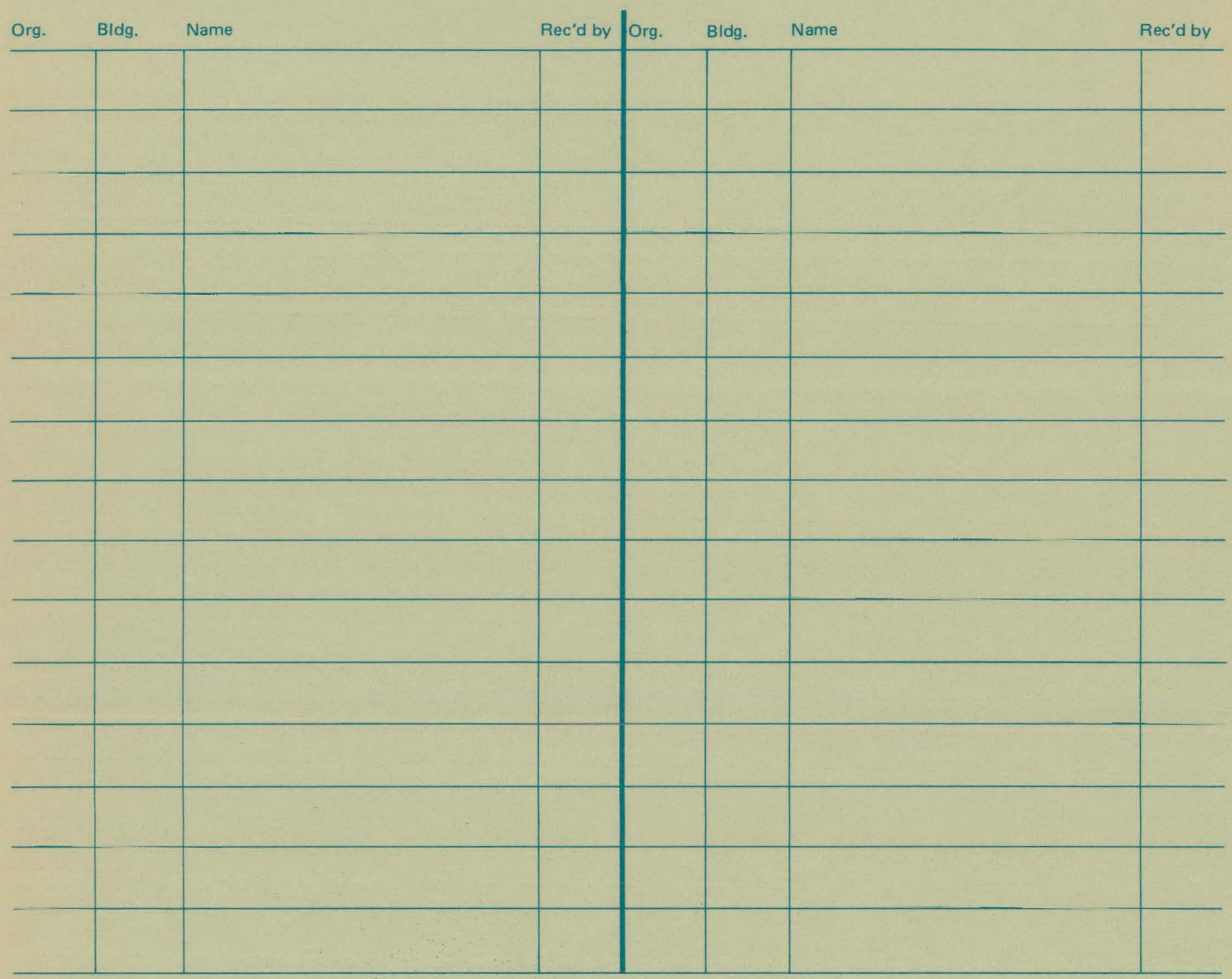

